\title{
Approximative Bayes Optimality Linear Discriminant Analysis for Chinese Handwriting Character Recognition
}

\author{
Chao Yao, Gong Cheng
}

Department of Control and Information, School of Automation, Northwestern Polytechnical University, 127 Youyi Xilu, Xi'an 710072, PR China

\begin{abstract}
Discriminant subspace learning is an important branch for pattern recognition and machine learning. Among the various methods, Bayes optimality linear discriminant analysis (BLDA) has shown its superiority both in theory and application. However, due to the computational complexity, BLDA has not been applied to large category pattern tasks yet. In this paper, we propose an approximative Bayes optimality linear discriminant analysis (aBLDA) method for Chinese handwriting character recognition, which is a typical large category task. In the aBLDA, we first select a set of convex polyhedrons that obtained by the state-of-the-art methods, then the searching zones are limited to these polyhedrons. Finally, the best of them is chosen as the final projection. In this way, the computational complexity of BLDA is reduced greatly with comparable accuracy. To find more than 1D projections, the orthogonal constraint is employed in the proposed method. The experimental results on synthetic data and CASIA-HWDB1.1 show the effectiveness of the proposed method.
\end{abstract}

Keywords: Dimension reduction, Bayes optimality, Chinese handwriting character recognition, large category, class separation problem.

\section{Introduction}

Extracting efficient feature from high-dimensional data is a crucial procedure in pattern classification tasks, for it could not only reduce the computational complexity, but also lead to better performance by removing the redundancy and noise of the data, meanwhile overcoming the curse-of-dimension problem for statistical classifier. In the past years, lots of methods have been proposed to solve the problem, such as Principal Component Analysis (PCA) [1], Linear Discriminant Analysis (LDA) [2], Independent Component Analysis (ICA) [3], Locality Preserving Projections (LPP) [4], kernel PCA [5], kernel LDA [6], ISOMAP [7], Locally Linear Embedding (LLE) [8] and so on. These methods could be categorized into linear and nonlinear ones based on d-

Email addresses: yaochao@nwpu.edu.cn (Chao Yao), chenggong1119@gmail .com (Gong Cheng) 
ifferent viewpoints, or supervised and unsupervised ones based on the samples' label information.

For large category pattern classification tasks, linear supervised feature extraction methods are widely employed. LDA, which aims to minimize the within class scatter and maximize the between class scatter at the same time in the low-dimensional space, is the most popular one among those methods. LDA only calculates the between scatter matrix and the within scatter matrix, then solves the eigenvalue decomposition problem, which makes LDA be implemented easily and computed efficiently. However, LDA would overemphasize on the classes that locate far-apart with each other for multi-class task. Various methods were developed to overcome the problem. Lotlikar et.al [9] introduced a fractional step method for the problem. In their method, a weighting function was used to increase the weights of the pairs that would be merged in fractional steps. Loog et.al [10] proposed another weighting function using the approximating of pairwise Bayes function. The function gave the pairs that locate nearly a large value according to Bayes rules. Recently, Zhang et.al [11] proposed a new weighting function based on the confusion matrix for the specific classifier. Nevertheless, these methods are not directly related to the classification error, and still have room for improvement in theoretical perspective.

Designing a Bayes optimal criterion for multi-class dimension reduction is a well known difficult job. However, progresses have been achieved under some constraint. For multi-class homoscedastic Gaussian distributed data with equal prior, Geisser [12] gave the formulation of Bayes error. Schervish [13] solved the 2D data for three classes which is a special case of [12]. Hamsici et.al [14] proposed a Bayes optimality linear discriminant analysis (BLDA). They first demonstrated the projections, which projected the means to the same order, formed a convex set. Therefore, the convex optimization methods could be used to solve the problem. To obtain the $2 \mathrm{D}$ or higher dimensional subspace, they iteratively found the 1D Bayes optimal projection in the orthogonal space. BLDA has shown its superiority to the other methods in various data sets. However, Hamsici claimed that $C ! / 2$ convex problems should be solved for each projection at maximum, where $C$ was the number of classes. With $C$ getting large, the number of convex spaces $C ! / 2$ increases sharply. Although they linearly approximated the Bayes error function to reduce the computational complexity, the performance of this approximation was not promising.

We focus on the handwriting Chinese character recognition (HCCR) task in this paper, which is a typical large category problem. For HCCR, LDA is a popular dimension reduction method. However, it might merge the similar characters (characters that looks alike) in the subspace, which degenerates the performance of HCCR. Methods on two aspects are proposed to solve this problem. On one hand, extra features are extracted for the similar characters. Leung et.al [15] extracted extra features for similar characters in the critical regions. Gao et.al [16] adopted LDA to obtain more discriminant feature for similar character pairs, then used a compound distance metric to classify the similar characters. Considering the similar characters were not only pair-wised, Wang et.al [17] extracted extra features for several similar characters simultaneously. In these methods, an extra classifier is employed to classify the similar characters, which leads to extra computational time and storage space. On the other hand, similar characters must be the classes locate nearly in the feature space. The 
aforementioned weighting methods could improve the separation problem of LDA. Zhang et.al [18] proved that the family of weighting LDA methods could improve the performance of HCCR, this is mainly because these methods assign large weights to the similar character pairs. However, to our best knowledge, the Bayes optimal methods have not been applied to HCCR or other large category pattern classification tasks yet due to their heavy computational complexity.

In this paper, we present an approximative BLDA (aBLDA) for HCCR. The main contribution of our work is that we reduce the searching spaces of BLDA significantly so that it could be used in HCCR task efficiently with comparable accuracy. In the proposed method, we do not search all the possible convex sets but those found by the popular linear dimension reduction methods, such as LDA, aPAC, the POW family and other methods that are already adopted by large category tasks. We first apply a set of dimension reduction methods on HCCR to get their individual 1D subspaces, and then use the 1D subspaces to find the convex sets determined by the above methods. Finally, the local Bayes optimality projections are found on these convex sets and the best of them is chosen as the final result. More projections could be obtained iteratively in the orthogonal space by our method. The proposed approximative BLDA is expected to have the following advantages: i) the number of convex sets that need to be searched is far less than BLDA; ii) with the improvement of Bayes optimality, the performance would be better than that of the existed ones. The experiments on the both synthetic data and CASIA-HWDB1.1 demonstrate the validity of the proposed method.

The rest of the paper is organized as following: the idea of BLDA is introduced in Section 2, then details of the approximative BLDA are presented in Section 3. We perform experiments in Section 4 to show the effectiveness of the proposed method and finally conclude it in Section 5.

\section{Review of Bayes Optimality in Linear Discriminant Analysis}

In this section, we will first introduce the details of BLDA [14]. For convenience, we present the important notations in the paper in Table 1. In the paper, Capital and lower letters in boldface denote matrix and vector. Matrix dimensions are shown as $(m \times n)$, where $m$ and $n$ are the numbers of rows and columns, respectively.

\subsection{The Bayes Optimal $1 D$ subspace}

Let $\mathbf{X} \in \mathbb{R}^{d \times n}$, where each row of $\mathbf{X}$ is a sample, be drawn from a set of homoscedastic Gaussian distributions with different means $\mu_{i}$ and equal prior probabilities, where $\boldsymbol{\Sigma}_{i}=\boldsymbol{\Sigma}, \forall i=1,2, \ldots, C$. Then the Bayes error when projecting the data on a single dimension can be formulated as:

$$
B_{\text {error }}=2 C^{-1} \sum \Phi\left(\frac{\eta_{(1)}-\eta_{(i+1)}}{2}\right)
$$

where $\Phi(\cdot)$ is the cumulative distribution function (cdf) of a standard Normal distribution, $\eta_{i}$ are the ordered projected and whitened means, where $\eta_{(1)}<\eta_{(2)}<\cdots<\eta_{(C)}$, and

$$
\eta_{i}=\frac{\mathbf{v}^{T} \mu_{i}}{\sqrt{\mathbf{v}^{T} \mathbf{\Sigma} \mathbf{v}}}=\mathbf{v}^{T} \hat{\mu}_{i}
$$


Table 1: Notations

\begin{tabular}{c||c}
\hline Notation & Description \\
\hline \hline $\mathbf{X}$ & data matrix \\
$\mu_{i}$ & centroid of the $i$ th class \\
$\mu$ & centroid of all samples \\
$\mathbf{S}_{b}$ & between-class scatter matrix \\
$\mathbf{\Sigma}_{i}$ & covariance matrix of the $i$ th class \\
$\mathbf{S}_{w}$ & within-class scatter matrix \\
$\mathbf{S}_{t}$ & total scatter matrix \\
$n$ & number of training samples \\
$n_{i}$ & number of training samples in the $i$ th class \\
$C$ & number of classes \\
$d$ & number of dimensions \\
$l$ & number of retained dimensions obtained by feature extraction \\
\hline
\end{tabular}

To solve the above problem, Hamsici proves that a constraint region $\mathcal{A}$, where all vectors $\mathbf{v}$ sampled from it generate the same ordered $\left\{\eta_{(i)}\right\}$, is a convex polyhedron, and the Bayes error function $B(\mathbf{v})$ is convex for $\forall \mathbf{v} \in \mathcal{A}$. Details of the proof could be found in [14]. In this way, the problem could be solved by searching all the convex regions and choosing the $\mathbf{v}_{\text {opt }}$ according to the minimal $B(\mathbf{v})$.

\subsection{Linear approximative BLDA}

In [14], a linear approximative BLDA (IBLDA) method is introduced. In the method, Eq.(1) is replaced by $\frac{1}{C} \sum a\left(\eta_{(i)}-\eta_{(i+1)}\right)+b$, then Eq.(1) becomes:

$$
B_{\text {error }}=-\frac{a\left(\eta_{(i)}-\eta_{(C)}\right)+(C-1) b}{C}
$$

For $\eta_{i}=\mathbf{v}^{T} \hat{\mu}_{i}$, where $\hat{\mu}_{i}$ is the whitening mean of the $i$ th class. The solution for Eq.(3) is $\mathbf{v}=\frac{\hat{\mu}_{i j}}{\left\|\hat{\mu}_{i j}\right\|}$, where $\hat{\mu}_{i j}$ is the maximal of the $C(C-1) / 2$ distances between the whitening means of each pair of classes. In this way, we just need to compute the $C(C-1) / 2$ distances and the computational complexity is far reduced.

\section{Approximative Bayes optimality Linear Discriminant Analysis}

We will discuss the obstacles for applying BLDA to the large category tasks here. To find 1D optimal Bayes projection, the convex problem defined in Eq.(1) should be solved for each constraint polyhedron. The number of the polyhedrons are related to the practical situation and its upper boundary is $C ! / 2$. To find $l$-dimensional data, the above procedure should be repeated $l$ times. For the large category problem, the class number $C$ is always very large, which makes the number of search spaces be extreme large. Thus, BLDA could not be used in such pattern recognition tasks directly.

In [14], a linear approximative method is presented, which is introduced in Section 2.2. However, the performance of the above approximative linear method is not 
promising according to the experimental results in [14]. The experimental results in HCCR task also prove this, which is presented in Section 4.2. Here, we solve the problem from another standpoint of view. In the practical, a number of dimension reduction methods are already adopted to solve the large category tasks. Comparing with all the possible convex polyhedrons, the number of the popular dimension methods is far small. When the convex spaces obtained by these popular methods are searched instead of the whole spaces, the resulting subspace is not always Bayes optimality but must be better than those obtained by the existing methods. In this way, the merit of BLDA could be employed in large category tasks with acceptable computational complexity. An example is illustrated in Fig.1. In this example, 4 classes in 2D whiten space are presented in Fig.1(a), where the crosses stand for the centroids and the circles stand for the variations of the data. In Fig.1(b), Bayes error in theory according to the projection is given according to Eq.(1). Here, we only search the convex areas that the existing methods locate and choose the one according to the minimum Bayes error as the final result. In this way, the result might not be the optimal projection, but it must be better than those existing ones theoretically. In this section, the details of the proposed method will be discussed.

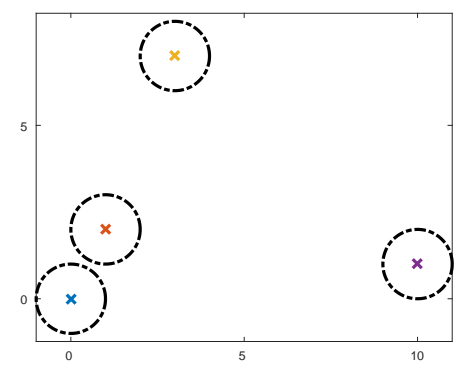

(a)

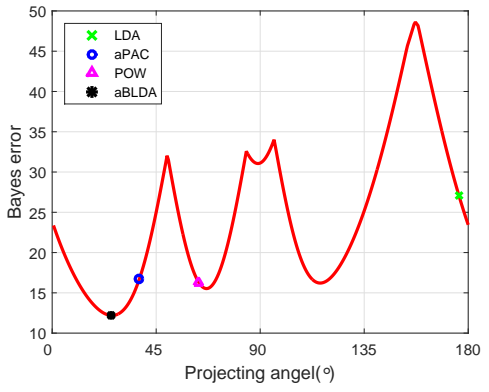

(b)

Figure 1: An toy example for aBLDA, (a) the toy data, (b) the Bayes error rate in 1-D space.

\subsection{Whitening}

As BLDA assumes the data are homoscedastic Gaussian distributed, the whitening procedure is first introduced. Given a set of data $\mathbf{X}=\left\{x_{1}, x_{2}, \cdots, x_{n}\right\} \in \mathbb{R}^{d \times n}$, the within-class scatter matrix $\mathbf{S}_{w}=\sum_{i=1}^{C} \sum_{j=1}^{n_{i}} p_{i}\left(\mathbf{x}_{i j}-\mu_{i}\right)\left(\mathbf{x}_{i j}-\mu_{i}\right)^{T}$, where $p_{i}$ is the prior probability of the $i$ th class and $\mu_{i}$ is the centroid of the $i$ th class. Let $\mathbf{P}$ and $\boldsymbol{\Lambda}$ be the eigenvector matrix and diagonal eigenvalue matrix of within-scatter matrix $\mathbf{S}_{w}$, respectively. So $\mathbf{S}_{w}=\mathbf{P} \Lambda \mathbf{P}^{T}$. Then the whitening transformation matrix $\mathbf{W}_{\text {whitening }}=\mathbf{P} \boldsymbol{\Lambda}^{-1 / 2}$. Thus, the whitened data $\hat{\mathbf{X}}$ are:

$$
\hat{\mathbf{X}}=\mathbf{W}_{\text {whitening }}^{T} \mathbf{X}_{i}
$$

In the whitening space, the within-scatter matrix $\hat{\mathbf{S}}_{w}=\mathbf{I}$. Assume $\mathbf{V}$ is the subspace obtained in the whitening space, the final subspace is:

$$
\mathbf{W}=\mathbf{W}_{\text {whitening }}^{T} \mathbf{V}
$$




\subsection{Selecting convex polyhedrons}

Various dimension reduction methods have been successfully adopted in large category pattern recognition tasks, we choose the following algorithms to provide the convex polyhedrons owing to their good performance, low complexity both in time and computation.

\subsection{1. $L D A$}

In the whitening space, LDA finds the projection that maximizes the distance between each class as follow:

$$
J(w)=\mathbf{w}^{T} \hat{\mathbf{S}}_{b} \mathbf{w}
$$

where the between-class scatter matrix $\hat{\mathbf{S}}_{b}=\sum_{i=1}^{C}\left(\hat{\mu}_{i}-\hat{\mu}\right)\left(\hat{\mu}_{i}-\hat{\mu}\right)^{T}$. The centroid of the $i$ th class is calculated as $\hat{\mu}_{i}=1 / n_{i} \sum_{k=1}^{n_{i}} \hat{\mathbf{x}}_{i k}$, the centroid of all the samples is $\hat{\mu}=1 / n \sum_{i=1}^{n} \hat{\mathbf{x}}_{i}$. The solution to Eq.(6) is the eigenvector corresponding to the largest eigenvalue of $\hat{\mathbf{S}}_{b}$.

\subsection{2. $a P A C$}

aPAC is introduced to solve the separation problem of LDA by employing a weighting scheme. In the method, the between-scatter matrix is modified to the following form:

$$
\hat{\mathbf{S}}_{b}^{a P A C}=\sum_{i=1}^{C-1} \sum_{j=i+1}^{C} \omega\left(\Delta_{i j}\right) p_{i} p_{j}\left(\hat{\mu}_{i}-\hat{\mu}_{j}\right)\left(\hat{\mu}_{i}-\hat{\mu}_{j}\right)^{T}
$$

where $\omega\left(\Delta_{i j}\right)=\frac{1}{2 \Delta_{i j}^{2}} \operatorname{erf}\left(\frac{\Delta_{i j}}{2 \sqrt{2}}\right), \Delta_{i j}=\sqrt{\left(\hat{\mu}_{i}-\hat{\mu}_{j}\right)^{T}\left(\hat{\mu}_{i}-\hat{\mu}_{j}\right)}$ and $\operatorname{erf}(x)=\frac{2}{\sqrt{\pi}} \int_{0}^{x} e^{-t^{2}} d t$ is the error function.

\subsubsection{Power function}

Power function is adopted in $[9,18]$ to calculate the weights between each pair of classes. The between-scatter matrix is defined as:

$$
\hat{\mathbf{S}}_{b}^{P O W}=\sum_{i=1}^{C-1} \sum_{j=i+1}^{C} f_{i j} p_{i} p_{j}\left(\hat{\mu}_{i}-\hat{\mu}_{j}\right)\left(\hat{\mu}_{i}-\hat{\mu}_{j}\right)^{T}
$$

where $f_{i j}=\left\|\hat{\mu}_{i}-\hat{\mu}_{j}\right\|_{2}^{-m}$ and $m$ is a positive integer. $f_{i j}$ is required to drop not slower than the Euclidean distance between the classes, so $m$ is suggested to be $m \geq 2$.

\subsubsection{Confused distance maximization}

Zhang et.al $[11,18]$ proposed a Confused distance maximization (CDM) method to solve the separation problem of LDA. The pair-wised weights in Eq.(8) are substituted by the confusion probabilities as following:

$$
f_{i j}=\left\{\begin{array}{l}
P_{i \rightarrow j}+P_{j \rightarrow i}, i \neq j \\
0, i=j
\end{array}\right.
$$


The confusion probability $P_{i \rightarrow j}$ is classifier based. It is computed on the training set by a predefined classifier using cross-validation or holdout-validation.

Besides the convex polyhedrons obtained by the mentioned methods, we also select a few convex zones randomly to enlarge the search space. In this way, the result of aBLDA is more likely to be the Bayes optimal. [14] proves that some sequences of projected centroid in $1 \mathrm{D}$ case are not available, so we generate some random projections and find their corresponding sequences of projected 1D centroid to obtain the random convex zones. Actually, the more convex zones are selected, the more accurate the result will be. At the same time, the longer training time it takes. The number of the convex polyhedrons should be limited in consideration of the computational complexity. In aBLDA, the total number of the convex zones is 50 .

\subsection{Optimization}

To get the Bayes optimality projection, we use Steepest Descent (SD) Method with constant step in each convex polyhedron to find the local minima. The basic SD method for Eq.(1) of the $i$ th convex polyhedron is

$$
\mathbf{v}_{k+1}^{i}=\mathbf{v}_{k}^{i}-\alpha \nabla \mathbf{B}\left(\mathbf{v}_{k}^{i}\right)
$$

where $\mathbf{v}_{0}^{i}$ is the result obtained by the other method, $\alpha$ is the step size, $\nabla \mathbf{B}(\mathbf{v})=$ $\left.-\frac{1}{C} \sum_{i=1}^{C-1} e^{-\frac{\mathbf{v}\left(\hat{\mu}_{i}-\hat{\mu}_{i+1}\right)}{2}}\left(\hat{\mu}_{i}-\hat{\mu}_{i+1}\right)\right)$. We set $\alpha$ with a small value to assure $\mathbf{v}^{i}$ do not jump to the other convex zones. In this way, we can get the optimal $\mathbf{v}^{i}$ with limited steps,.

\subsection{Approximative Bayes Optimal More than $1 D$ subspace}

To find the subspace more than $1 \mathrm{D}$, a constraint is always applied between the projecting vectors. Orthogonal (or named Foley-Sammon) [19, 20] and uncorrelated $[21,22,23]$ are two typical constraints which have been well studied and widely used in many areas. In this paper, we follow the orthogonal constraint which is defined as:

$$
\left\{\begin{array}{l}
\mathbf{w}_{i}^{T} \mathbf{w}_{j}=0, i \neq j \\
\left\|\mathbf{w}_{i}\right\|_{2}=1
\end{array}\right.
$$

To constrain the subspace to be orthogonal, we give the following theorems without proof.

Theorem 1. For space $\mathbf{V}_{k}=\left(\mathbf{v}_{1}, \mathbf{v}_{2}, \ldots, \mathbf{v}_{k}\right)$, where $\mathbf{v}_{i}^{T} \mathbf{v}_{j}=\delta_{i j}$, then its orthogonal space is spanned by the eigenvectors of $P=I-\mathbf{V}_{k} \mathbf{V}_{k}^{T}$ with their corresponding eigenvalues being 1 .

Theorem 2. $\mathbf{v}_{k+1}^{\prime} \in \mathbf{V}_{k}^{\perp}$, thus $\mathbf{v}_{k+1}=\mathbf{V}_{k} \mathbf{v}_{k+1}^{\prime}$ is the $(k+1)$ th discriminant vector that satisfies the orthogonal constraint.

With Theorem1 and Theorem2, the details of finding $d$-dimensional subspace are as following. Suppose we have the $k$-dimensional subspace $\mathbf{V}_{k}=\left(\mathbf{v}_{1}, \mathbf{v}_{2}, \ldots, \mathbf{v}_{k}\right)$, then we project the mean vectors to the orthogonal space by the method in Theorem1, and find the 1D Bayes optimal vector. Afterward, we project the obtained vector back to the original space by Theorem 2 to get the $(k+1)$ th projecting vector. Finally, the $(k+1)$ th subspace is $\mathbf{V}_{k+1}=\left(\mathbf{V}_{k}, \mathbf{v}_{k+1}\right)$. 


\subsection{Algorithm}

In aBLDA, we first solve the algorithms in section 3.2 to confirm the convex polyhedrons, then Bayes optimality projections are founded in these polyhedrons and the best one among these projections is chosen, which is denoted as $\mathbf{v}_{1}$. To find the second projection, the data are projected to $\mathbf{v}_{1}$ 's orthogonal subspace $\mathbf{V}_{1}^{\perp}$. By repeating the same algorithm in $\mathbf{V}_{1}^{\perp}$, we can find the second projection $\mathbf{v}_{2}$ '. With Theorem 2, the second projection $\mathbf{v}_{2}$ is obtained. Denoting $\mathbf{V}_{2}=\left(\mathbf{v}_{1}, \mathbf{v}_{2}\right)$, and $\mathbf{v}_{1}^{T} \mathbf{v}_{2}=0$. To find a subspace with l-dimensional, we can recursively apply the above procedure. The algorithm of aBLDA is summarized in Algorithm 1.

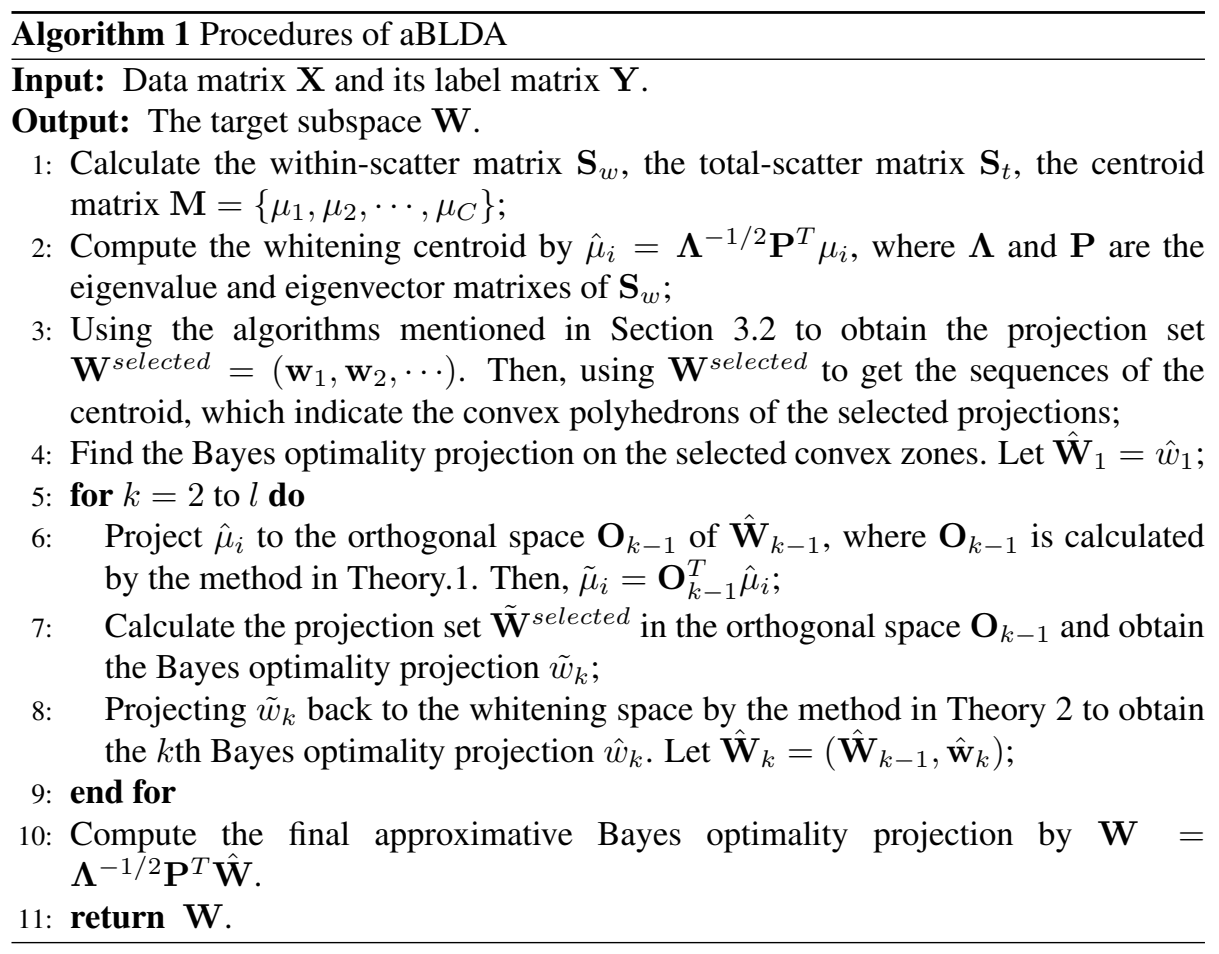

\section{Experiments}

In the experiments, we evaluate the proposed method on synthetic data and CASIAHWDB1.1, a public Chinese handwriting character data set with 3755 classes. All the subspace learning algorithms are programmed in MATLAB2015 and executed on a PC(CPU: Inter i5 3.30GHz, RAM: 8GB).

\subsection{A study on the approximative capability}

To compare the performance of aBLDA with BLDA, we first experiment on the synthetic data. In this test, the synthetic data are sampled from several homoscedastic Gaussian distributions. As aBLDA searches a part of the convex polyhedrons, the ratio 
Table 2: The classification results in 1D subspace for the synthetic data

\begin{tabular}{ccccc}
\hline number of class & 4 & 5 & 6 & 7 \\
\hline LDA & $84.17 \pm 0.93$ & $76.19 \pm 0.90$ & $70.57 \pm 0.81$ & $64.68 \pm 0.62$ \\
aPAC & $88.06 \pm 0.96$ & $81.75 \pm 0.80$ & $76.00 \pm 0.65$ & $69.80 \pm 0.60$ \\
POW3 & $86.69 \pm 1.01$ & $80.89 \pm 0.80$ & $75.60 \pm 0.67$ & $69.69 \pm 0.58$ \\
lBLDA & $90.55 \pm 0.60$ & $81.80 \pm 0.67$ & $74.80 \pm 0.59$ & $68.42 \pm 0.49$ \\
aBLDA & $99.01 \pm 0.01$ & $94.85 \pm 0.06$ & $88.02 \pm 0.13$ & $80.01 \pm 0.18$ \\
BLDA & $99.05 \pm 0.01$ & $95.15 \pm 0.05$ & $88.40 \pm 0.11$ & $80.28 \pm 0.14$ \\
\hline
\end{tabular}

between the searched zones and the whole set must influence aBLDA's performance. We vary the number of Gaussian distributions with 4, 5, 6 and 7 in the experiments. For each Gaussian distribution $N_{i}\left(\mu_{i}, \boldsymbol{\Sigma}_{i}\right)$, the centroid $\mu_{\mathbf{i}}$ is sampled from a uniformly distributed cube, whose $r=10$. The covariance matrix is set to be $\boldsymbol{\Sigma}_{i}=\mathbf{I}$ to simulate the whitened data. The dimensionality of the data is $d=12$ for all synthetic data. We use 500 samples for training and 100 samples for testing in each class and repeat the process for 1000 times.

Besides BLDA, we also compare aBLDA with LDA, POW3, aPAC, and 1BLDA, which provide the convex zones for aBLDA in the experiments. Here, POW3 denotes the power function in section 3.2.3 with $m=3$ in Eq.(8). The Nearest Class Mean (NCM) classifier, which is proved to be optimal under homoscedastic Gaussian assumption, is adopted in this test. NCM is based on Euclidean distance and could be denoted as:

$$
y_{\mathbf{x}}^{N C M}=\arg \min _{i}\left\|\mathbf{x}-\mu_{i}\right\|_{2}^{2}
$$

The results are presented in Fig.2. From the experimental results, BLDA and aBLDA perform much better than the other methods in the low dimensional cases, and aBLDA is slightly worse than BLDA. The classification accuracies and derivations of each algorithm in 1D subspace are listed in Table 2. We also present the consuming time of each algorithm in Fig.3. From the result, we can see that the computational time of BLDA increases dramatically with the growing of class number, but the consuming time of aBLDA increases slightly. This is mainly because aBLDA only searches 4 convex areas for each experiment, and BLDA searches 12, 60, 360 and 2520 convex areas, respectively. It proves that the proposed aBLDA improves the computation efficiency of BLDA significantly with slightly loss of accuracy.

\subsection{Experiments on CASIA-HWDB1.1}

We test aBLDA on a real-world large category data set, CASIA-HWDB1.1 [24], in this subsection. The CASIA-HWDB1.1 is a public Chinese handwriting character data set which contains 897758 samples for training and 223991 samples for testing. The samples are from the most frequently used 3755 classes (GB2312-80 level 1) that cover 99.2\% daily use in mainland of China. A part of the samples are demonstrated in Fig.4. The normalized-gradient features are extracted from the gray scale character images, where the gradient is quantized into eight directions for each pixel, then the histograms of the gradients are obtained from $8 \times 8$ grids. In this way, a 512-dimensional feature 


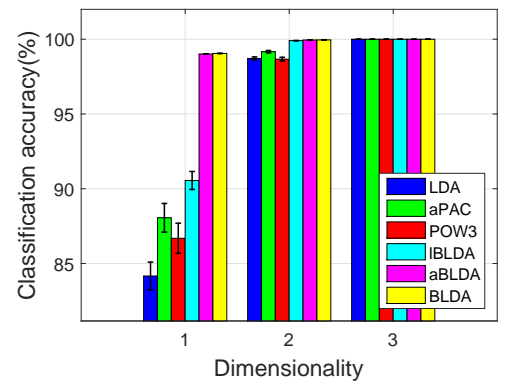

(a)

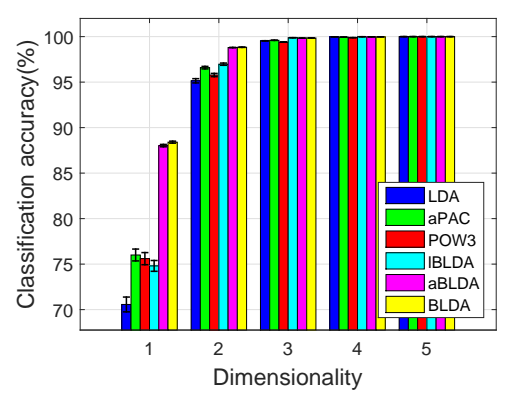

(c)

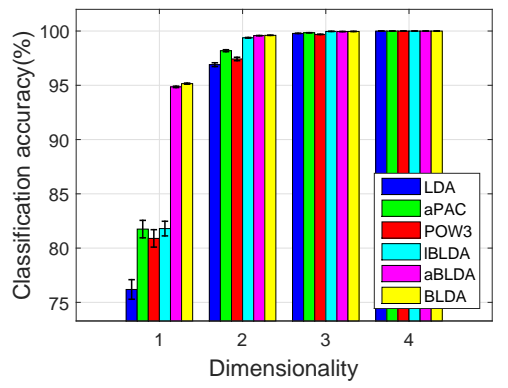

(b)

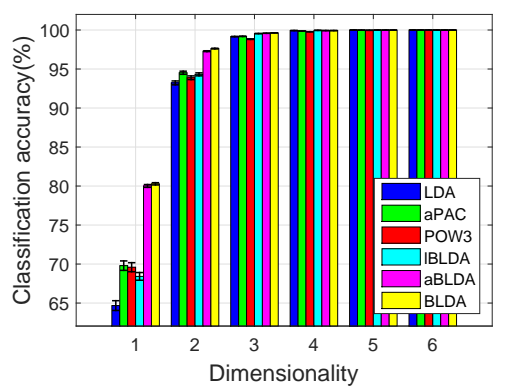

(d)

Figure 2: Average and standard deviations classification accuracies on synthetic data. (a) 4 classes, (b) 5 classes, (c) 6 classes, (d) 7 classes.

vector is formed to represent each character image. The feature data are available from [25]. Noting that the Box-Cox [26] transformation is performed with $m=0.5$.

In this test, we compare aBLDA with PCA [1], LDA [2], aPAC [10], POW2, POW3, POW5, POW7, POW10 [9], CDM [18] and IBLDA [14] which provide the search convex zones for aBLDA. POW2, POW3, POW5, POW7 and POW10 mean the different powers of Eq.(8). The CDM for Chinese handwriting character task is also compared in our experiments.

Besides NCM classifier, we also adopt MQDF [27] classifier in this test. Based on the Bayes decision rule, which classifies the input to the class with the maximum a posterior probability. Quadratic Discriminant Function (QDF) [28] is proposed under the assumption of Gaussian density for each class. MQDF improves the computation efficiency and accuracy of QDF by smoothing the minor eigenvalue of each class' covariance matrix. MQDF is a state-of-the-art classifier that has been widely used in HCCR, so we use it to evaluate the performances of the aforementioned methods. The MQDF is derived from QDF, which is:

$$
y_{\mathbf{x}}^{Q D F}=\arg \min _{i}\left(\mathbf{x}-\mu_{i}\right)^{T} \boldsymbol{\Sigma}_{i}^{-1}\left(\mathbf{x}-\mu_{i}\right)-\log \left|\boldsymbol{\Sigma}_{i}\right|
$$

Kimura et.al find that the minor eigenvalues of $\boldsymbol{\Sigma}_{i}$ is more sensitive to the estimation error, so they replace these minor eigenvalues with a constant to improve the 


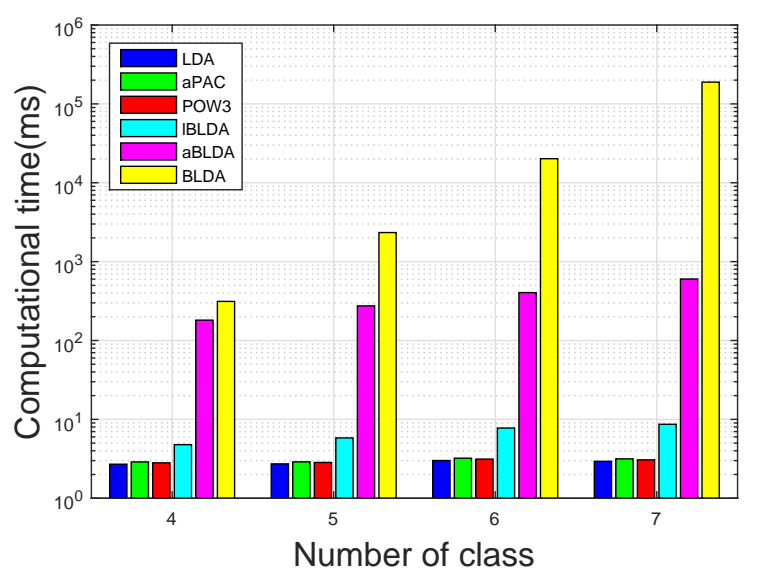

Figure 3: Computational time for synthetic data.

performance of QDF. The MQDF could be denoted as:

$y_{\mathbf{x}}^{M Q D F}=\arg \min _{i} \frac{1}{h_{i}}\left(\left\|\mathbf{x}-\mu_{i}\right\|_{2}^{2}-\sum_{j=1}^{k}\left(1-\frac{h_{i}}{\lambda_{i j}}\right) \phi_{i j}^{T}\left(\mathbf{x}-\mu_{i}\right)^{2}\right)+\sum_{j=1}^{k} \log \lambda_{i j}+(d-k) \log h_{i}$

where $\lambda_{i j}$ and $\phi_{i j}$ are the $j$ th largest eigenvalue and its corresponding eigenvector of $\Sigma_{i}$, respectively. $k$ is the number of principal eigenvectors that kept in MQDF. $h_{i}$ replaces the minor eigenvalues $\lambda_{i(k+1)}, \ldots, \lambda_{i d}$. It can be set to a class-independent constant or class-dependent constant. Here we set $h_{i}$ to be class-independent for its superior performance [29].

In the experiment, we divide the training set into two subsets for CDM, where $3 / 4$ of the samples for training the NCM and the other $1 / 4$ samples for estimating the confusion matrix. The performances of each algorithms are evaluated with the classification accuracy when the dimensionality of the subspace $l=50,60, \ldots, 180$. For MQDF, we set $k=50$ empirically for all methods, and search the value of $h_{i}$ on the training set. To speed up MQDF, we first use NCM to provide 100 candidates in the experiments. The experimental results are shown in Table 3 and Table 4.

From the experimental results, we see that aBLDA outperforms the other methods in most cases. It proves the effectiveness of aBLDA in large category classification task. aBLDA performs slightly worse than the other methods in several cases, it might because that the homoscedastic assumption is not satisfied. Noting the significant improvement of aBLDA at the low-dimensional subspaces, such as $l=\{50,60,70\}$. It makes aBLDA very suitable for the applications with limitation to memory and computation power, e.g. the smart phone, tablet PC.

To investigate why aBLDA can outperform the other methods in the lower-dimensional situations, we compute the Euclidean distances between some similar character pairs when $l=60$. The results are list in Fig.4. The results show that the confusion classes locate more distantly in the subspace learnt by aBLDA than the ones learnt by oth- 
呵埃换嗼唉哀㿠癌蔼矮艾碍爱险㛺安俺按暗胺 肮盎四敖謷奥澳芭捌扒叭吧笆入疤巴拔咙靶把 耙坝罢爸白柏百摆伯败拜斑班扳颁板版扮拌伴 半办绊邦梆膀绑磅谤苞胞包襄剩薄保饱宝的杯 悲北背贝钦倍顷焙被莱本策煳柔蹋迸比彼蔽毕

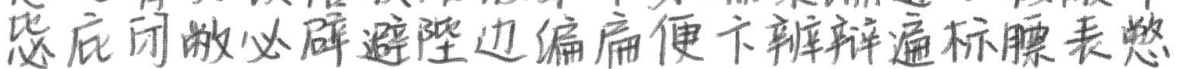
别柇瑸宾摈兵冰柄丙饼炳病并玻菠播钵波搏铂 伯舶牸膊勃泊玫捕上哺补不怖裁材才财睬采彩

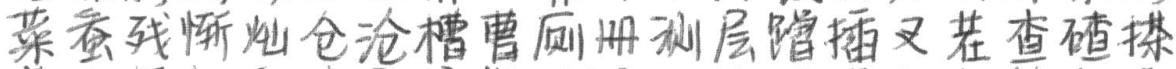
差柁拆柴豺说昌场尝长肠厂敞畅唱倡超抄朝潮 吵车扯彻澈脚臣辰尘忱沉陈趁衬称橙成呈乘程 復澄诚承道吃持匙池迟驰驰耻侈尺赤翅有炽虫

Figure 4: Some samples of Chinese characters in CASIA-HWDB1.1

er methods in most cases. Thus, aBLDA is more discriminative to the classes locate closely than other methods.

\section{Conclusion}

This paper presents an approximative Bayes optimality dimension reduction method for large category classification task. In the proposed method, a set of convex polyhedrons are computed firstly. Then, the Bayes optimality projections are founded among these polyhedrons. Finally, the best one among these Bayes optimality projections is selected as the solution. More projections are obtained in the orthogonal space of the previous solution. To our best knowledge, it is the first attempt to bring Bayes optimality subspace to handwriting Chinese character classification task. The experimental results on synthetic data and the handwriting Chinese characters show the efficiency of the proposed method both in time and accuracy.

In the practical, the distribution of the data could not always meet the homoscedastic assumption for the Bayes optimality projection, which will degenerate the performance of the proposed method. Kernel tricks could be adopted to relax the homoscedastic assumption. However, applying kernel method to large category tasks is very difficult. We will work on how to relax the homoscedastic assumption on the large category tasks, such as remote sensing image classification [30, 31, 32, 33, 34], object recognition [35, 36, 37] and biological images [38, 39, 40], in our future works. 
Table 3: Classification accuracies of different dimensionality with NCM

\begin{tabular}{cccccccccccc}
\hline$l$ & PCA & LDA & aPAC & POW2 & POW3 & POW5 & POW7 & POW10 & CDM & lBLDA & aBLDA \\
\hline 50 & 67.04 & 77.11 & 77.19 & 77.31 & 77.44 & 77.52 & 77.58 & 77.61 & 77.57 & 61.82 & $\mathbf{7 8 . 0 1}$ \\
60 & 69.84 & 78.80 & 78.90 & 78.94 & 79.00 & 79.17 & 79.22 & 79.18 & 78.95 & 66.85 & $\mathbf{7 9 . 9 6}$ \\
70 & 71.76 & 79.88 & 80.00 & 80.08 & 80.12 & 80.20 & 80.23 & 80.24 & 79.96 & 70.16 & $\mathbf{8 1 . 0 3}$ \\
80 & 73.07 & 80.56 & 80.62 & 80.72 & 80.80 & 80.86 & 80.91 & 80.92 & 80.63 & 72.65 & $\mathbf{8 1 . 0 5}$ \\
90 & 73.80 & 81.07 & 81.12 & 81.18 & 81.20 & 81.30 & $\mathbf{8 1 . 3 5}$ & 81.31 & 81.09 & 74.40 & 81.11 \\
100 & 74.58 & 81.43 & 81.47 & 81.53 & 81.55 & 81.62 & 81.63 & 81.64 & 81.44 & 75.72 & $\mathbf{8 1 . 7 8}$ \\
110 & 74.84 & 81.71 & 81.75 & 81.76 & 81.80 & 81.86 & 81.89 & 81.90 & 81.66 & 76.78 & $\mathbf{8 2 . 0 0}$ \\
120 & 75.29 & 81.88 & 81.91 & 81.96 & 81.94 & 81.99 & 82.02 & 82.03 & 81.83 & 77.60 & $\mathbf{8 2 . 0 7}$ \\
130 & 75.51 & 81.98 & 82.00 & 82.01 & 82.02 & 82.06 & 82.12 & $\mathbf{8 2 . 1 3}$ & 82.02 & 78.33 & 82.09 \\
140 & 75.70 & 82.09 & 82.07 & 82.06 & 82.08 & 82.13 & $\mathbf{8 2 . 1 6}$ & 82.11 & 82.04 & 78.86 & 82.13 \\
150 & 75.87 & 82.12 & 82.14 & 82.16 & 82.16 & 82.16 & 82.17 & 82.18 & 82.10 & 79.26 & $\mathbf{8 2 . 1 9}$ \\
160 & 76.00 & 82.14 & 82.15 & 82.16 & 82.16 & 82.22 & 82.18 & 82.20 & 82.11 & 79.70 & $\mathbf{8 2 . 2 3}$ \\
170 & 76.09 & 82.16 & 82.16 & 82.17 & 82.18 & 82.16 & 82.19 & 82.17 & 82.12 & 80.00 & $\mathbf{8 2 . 2 3}$ \\
180 & 76.17 & 82.19 & 82.19 & 82.19 & 82.20 & 82.16 & 82.20 & 82.19 & 82.16 & 80.20 & $\mathbf{8 2 . 2 7}$ \\
average & 73.97 & 81.08 & 81.12 & 81.16 & 81.19 & 81.24 & 81.27 & 81.27 & 81.12 & 75.17 & $\mathbf{8 1 . 4 4}$ \\
\hline
\end{tabular}

Table 4: Classification accuracies of different dimensionality with MQDF

\begin{tabular}{cccccccccccc}
\hline$l$ & PCA & LDA & aPAC & POW2 & POW3 & POW5 & POW7 & POW10 & CDM & lBLDA & aBLDA \\
\hline 50 & 71.03 & 80.85 & 80.88 & 80.85 & 80.91 & 81.16 & 81.95 & 82.35 & 81.87 & 69.58 & $\mathbf{8 3 . 4 7}$ \\
60 & 73.12 & 82.75 & 82.73 & 82.76 & 82.83 & 82.98 & 83.02 & 84.26 & 82.97 & 70.23 & $\mathbf{8 5 . 2 3}$ \\
70 & 74.36 & 84.48 & 84.59 & 84.68 & 84.75 & 84.79 & 84.82 & 84.75 & 84.61 & 74.02 & $\mathbf{8 6 . 1 7}$ \\
80 & 76.01 & 85.94 & 86.03 & 86.22 & 86.23 & 86.02 & 86.26 & 85.59 & 86.14 & 75.89 & $\mathbf{8 7 . 0 2}$ \\
90 & 76.92 & 87.11 & 87.35 & 86.98 & 87.19 & 87.27 & 87.13 & $\mathbf{8 7 . 3 2}$ & 87.22 & 77.25 & 87.29 \\
100 & 77.56 & 87.88 & 87.74 & 87.59 & 87.95 & 88.13 & $\mathbf{8 8 . 1 6}$ & 87.79 & 87.92 & 79.03 & 88.12 \\
110 & 78.22 & 88.46 & 88.29 & 88.31 & 88.48 & 88.87 & 88.79 & 87.99 & 88.25 & 79.94 & $\mathbf{8 8 . 9 4}$ \\
120 & 78.81 & 88.89 & 89.02 & 89.13 & 89.20 & $\mathbf{8 9 . 5 2}$ & 89.25 & 89.21 & 88.89 & 80.35 & 88.97 \\
130 & 80.12 & 89.18 & 89.12 & 89.19 & 89.32 & 89.53 & 89.47 & 89.32 & 89.12 & 81.89 & $\mathbf{8 9 . 5 4}$ \\
140 & 80.76 & 89.38 & 89.34 & 89.40 & 89.39 & 89.55 & $\mathbf{8 9 . 5 7}$ & 89.42 & 89.15 & 82.45 & 89.55 \\
150 & 80.88 & 89.45 & 89.39 & 89.48 & 89.47 & 89.52 & 89.54 & 89.41 & 89.19 & 83.06 & $\mathbf{8 9 . 5 9}$ \\
160 & 81.32 & 89.50 & 89.41 & 89.51 & 89.51 & 89.55 & 89.55 & 89.35 & 89.28 & 84.21 & $\mathbf{8 9 . 6 2}$ \\
170 & 82.01 & 89.49 & 89.46 & 89.51 & 89.52 & 89.57 & 89.57 & 89.41 & 89.35 & 84.20 & $\mathbf{8 9 . 6 3}$ \\
180 & 82.05 & 89.49 & 89.50 & 89.53 & 89.53 & 89.56 & 89.58 & 89.40 & 89.37 & 84.21 & $\mathbf{8 9 . 6 5}$ \\
average & 78.08 & 87.35 & 87.35 & 87.37 & 87.45 & 87.57 & 87.62 & 87.54 & 87.38 & 79.02 & $\mathbf{8 8 . 0 6}$ \\
\hline
\end{tabular}

\section{Acknowledgments}

We would like to thank the associate editor and all anonymous reviewers for their constructive comments and suggestions. This work was partially supported by the National Science Foundation of China under Grant 61401357 and the Aerospace Science Foundation of China under Grant 20140153003.

\section{References}

[1] I. Jolliffe, Principal component analysis, Wiley Online Library, 2002.

[2] R. Fisher, The use of multiple measurements in taxonomic problems, Annals of Human Genetics 7 (2) (1936) 179-188.

[3] A. Hyvärinen, J. Karhunen, E. Oja, Independent component analysis, Vol. 46, John Wiley \& Sons, 2004. 


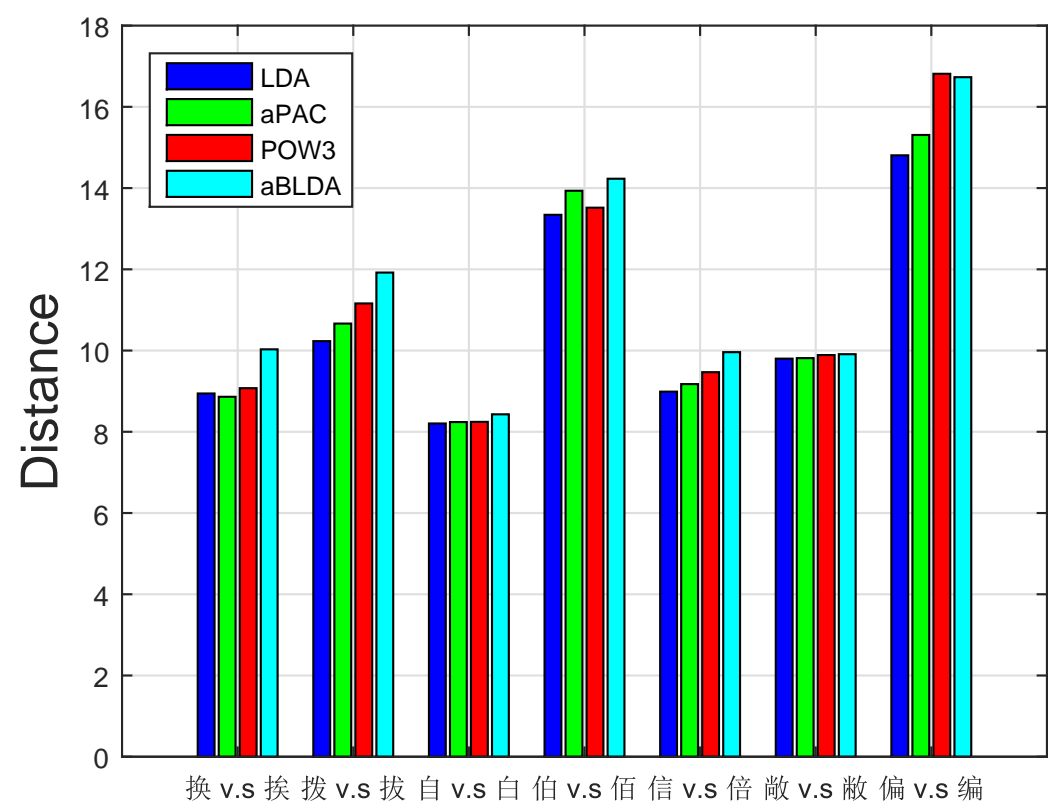

Figure 5: Comparison of distances between confusion characters in different subspaces

[4] X. Niyogi, Locality preserving projections, in: Neural information processing systems, Vol. 16, MIT, 2004, p. 153.

[5] B. Schölkopf, A. Smola, K.-R. Müller, Kernel principal component analysis, in: Artificial Neural NetworksłICANN'97, Springer, 1997, pp. 583-588.

[6] B. Schölkopf, A. Smola, K.-R. Müller, Nonlinear component analysis as a kernel eigenvalue problem, Neural computation 10 (5) (1998) 1299-1319.

[7] J. B. Tenenbaum, V. De Silva, J. C. Langford, A global geometric framework for nonlinear dimensionality reduction, Science 290 (5500) (2000) 2319-2323.

[8] S. T. Roweis, L. K. Saul, Nonlinear dimensionality reduction by locally linear embedding, Science 290 (5500) (2000) 2323-2326.

[9] R. Lotlikar, R. Kothari, Fractional-step dimensionality reduction, Pattern Analysis and Machine Intelligence, IEEE Transactions on 22 (6) (2000) 623 -627.

[10] M. Loog, R. Duin, R. Haeb-Umbach, Multiclass linear dimension reduction by weighted pairwise fisher criteria, Pattern Analysis and Machine Intelligence, IEEE Transactions on 23 (7) (2001) 762-766. 
[11] X.-Y. Zhang, C.-L. Liu, Confused distance maximization for large category dimensionality reduction, in: Frontiers in Handwriting Recognition (ICFHR), 2012 International Conference on, IEEE, 2012, pp. 213-218.

[12] S. Geisser, Discrimination, allocatory and separatory, linear aspects, Tech. rep., DTIC Document (1976).

[13] M. Schervish, Linear discrimination for three known normal populations, Journal of statistical planning and inference 10 (2) (1984) 167-175.

[14] O. C. Hamsici, A. M. Martinez, Bayes optimality in linear discriminant analysis, Pattern Analysis and Machine Intelligence, IEEE Transactions on 30 (4) (2008) 647-657.

[15] K. Leung, C. Leung, Recognition of handwritten chinese characters by critical region analysis, Pattern Recognition 43 (3) (2010) 949-961.

[16] T.-F. Gao, C.-L. Liu, High accuracy handwritten chinese character recognition using lda-based compound distances, Pattern Recognition 41 (11) (2008) 34423451 .

[17] Y. Wang, C. Liu, X. Ding, Similar pattern discriminant analysis for improving chinese character recognition accuracy, in: Document Analysis and Recognition (ICDAR), 2013 12th International Conference on, IEEE, 2013, pp. 1056-1060.

[18] X.-Y. Zhang, C.-L. Liu, Evaluation of weighted fisher criteria for large category dimensionality reduction in application to chinese handwriting recognition, Pattern recognition 46 (9) (2013) 2599-2611.

[19] D. H. Foley, J. W. Sammon, An optimal set of discriminant vectors, Computers, IEEE Transactions on 100 (3) (1975) 281-289.

[20] W. Zheng, L. Zhao, C. Zou, Foley-sammon optimal discriminant vectors using kernel approach, Neural Networks, IEEE Transactions on 16 (1) (2005) 1-9.

[21] J. Ye, R. Janardan, Q. Li, H. Park, Feature reduction via generalized uncorrelated linear discriminant analysis, Knowledge and Data Engineering, IEEE Transactions on 18 (10) (2006) 1312-1322.

[22] W.-H. Yang, D.-Q. Dai, H. Yan, Feature extraction and uncorrelated discriminant analysis for high-dimensional data, Knowledge and Data Engineering, IEEE Transactions on 20 (5) (2008) 601-614.

[23] C. Yao, Z. Lu, J. Li, W. Jiang, J. Han, An improved fisher discriminant vector employing updated between-scatter matrix, Neurocomputing 173 (2016) 154162.

[24] C.-L. Liu, F. Yin, D.-H. Wang, Q.-F. Wang, Casia online and offline chinese handwriting databases, in: Document Analysis and Recognition (ICDAR), 2011 International Conference on, IEEE, 2011, pp. 37-41. 
[25] http://www.nlpr.ia.ac.cn/databases/handwriting/Home. html.

[26] R. Sakia, The box-cox transformation technique: a review, The statistician (1992) 169-178.

[27] F. Kimura, K. Takashina, S. Tsuruoka, Y. Miyake, Modified quadratic discriminant functions and the application to chinese character recognition, Pattern Analysis and Machine Intelligence, IEEE Transactions on PAMI-9 (1) (1987) 149-153.

[28] R. O. Duda, P. E. Hart, D. G. Stork, Pattern classification (2nd edition), En Broeck the Statistical Mechanics of Learning Rsity.

[29] T. Long, L. Jin, Building compact mqdf classifier for large character set recognition by subspace distribution sharing, Pattern Recognition 41 (9) (2008) 29162925.

[30] J. Han, P. Zhou, D. Zhang, G. Cheng, L. Guo, Z. Liu, S. Bu, J. Wu, Efficient, simultaneous detection of multi-class geospatial targets based on visual saliency modeling and discriminative learning of sparse coding, ISPRS Journal of Photogrammetry and Remote Sensing 89 (2014) $37-48$.

[31] G. Cheng, J. Han, P. Zhou, L. Guo, Multi-class geospatial object detection and geographic image classification based on collection of part detectors, ISPRS Journal of Photogrammetry and Remote Sensing 98 (2014) 119-132.

[32] G. Cheng, J. Han, L. Guo, Z. Liu, S. Bu, J. Ren, Effective and efficient midlevel visual elements-oriented land-use classification using vhr remote sensing images, Geoscience and Remote Sensing, IEEE Transactions on 53 (8) (2015) 4238-4249.

[33] G. Cheng, P. Zhou, J. Han, L. Guo, Auto-encoder-based shared mid-level visual dictionary learning for scene classification using very high resolution remote sensing images, IET Computer Vision 9 (5) (2015) 639-647.

[34] P. Zhou, G. Cheng, Z. Liu, S. Bu, X. Hu, Weakly supervised target detection in remote sensing images based on transferred deep features and negative bootstrapping, Multidimensional Systems and Signal Processing (2015) doi:10.1007/s11045-015-0370-3.

URL http://dx.doi.org/10.1007/s11045-015-0370-3

[35] J. Han, D. Zhang, G. Cheng, L. Guo, J. Ren, Object detection in optical remote sensing images based on weakly supervised learning and high-level feature learning, Geoscience and Remote Sensing, IEEE Transactions on 53 (6) (2015) 33253337.

[36] J. Han, D. Zhang, X. Hu, L. Guo, J. Ren, F. Wu, Background prior-based salient object detection via deep reconstruction residual, IEEE Transactions on Circuits and Systems for Video Technology 25 (8) (2015) 1309-1321. 
[37] J. Han, S. He, X. Qian, D. Wang, L. Guo, T. Liu, An object-oriented visual saliency detection framework based on sparse coding representations, Circuits and Systems for Video Technology, IEEE Transactions on 23 (12) (2013) 2009-2021.

[38] J. Han, X. Ji, X. Hu, D. Zhu, K. Li, X. Jiang, G. Cui, L. Guo, T. Liu, Representing and retrieving video shots in human-centric brain imaging space, Image Processing, IEEE Transactions on 22 (7) (2013) 2723-2736.

[39] J. Han, C. Chen, L. Shao, X. Hu, Learning computational models of video memorability from fmri brain imaging, IEEE Transactions on Cybernetics 45 (8) (2015) 1692-1703.

[40] J. Han, D. Zhang, S. Wen, L. Guo, T. Liu, X. Li, Two-stage learning to predict human eye fixations via sdaes, Cybernetics, IEEE Transactions on 46 (2) (2016) 487-498. 